\title{
The Research of Simulation Design of Non-metallic Bulletproof Helmet
}

\author{
Haixin Jin and Jiangchang Jin
}

\begin{abstract}
In the paper, the non-metallic bulletproof helmet is analyzed in research. The way can not accurately describe and transmit the shape messages of free-curve and free-camber in design drawing by hand, design period long and a large amount of work, a large number of data are lose and man-made muff. Advance to use the way combine Rhino software and AutoCAD software in The Computer Simulation Auxiliary Design of non-metallic bulletproof helmet, raise design level.
\end{abstract}

Index Terms-Rhino, non-metallic bulletproof helmet, simulation design, auto CAD.

\section{INTRODUCTION}

Since the beginning of the 21 st century, computer technology, the CAD technology in particular, plays an increasingly important role in the product design industry. It has brought fundamental changes to the traditional design, manufacturing and production management and is provided with prominent economic benefits and huge potential for development. Military non-metal bulletproof helmet is composed by high-performance fiber fabric and its traditional design is the manual operation by using a pen drawing on drawing paper. The show is a plan. While the use of computer simulation-aided design is a three-dimensional modeling process and the show is an electronic model. Computer-aided design can not be separated from design software. Currently there is a wide variety of $3 \mathrm{D}$ design software, so we can use it freely or in combination according to our need. If we use the Rhino software, what we need is just simply entering the relevant data and soon it will be able to generate three-dimensional stereoscopic graphics in perspective and show the design work flexibly [1], [2].

Rhino modeling capabilities are very powerful and easy to read and they are great masters of NURBS (Non-Uniform Rational B Spline) curve shape. They loft the formation of surface easily through a series of fully closed or not closed curve, as well as make the curve along the axis of rotational molding surfaces or through the curve one path sweep or two paths sweep into the surface easily. It is more convincing when generated to cover the surface and generate the surface by the color values of each pixel in the bitmap. Moreover, it is also the first set of software which introduces AGHB NURBS modeling technology to the Windows operating

Manuscript received December 1, 2014; revised April 30, 2015.

Haixin Jin is with the Academy of Arts \& Design, Tsinghua University, Beijing 100084, China (e-mail: jinhaixin911@126.com).

Jiangchang Jin was with the Huazhong University of Science and Technology, Hubei, WH430074, China. He is now with the Test Institute of Quality \& Measurement, Guangdong, Shenzhen 518000, China (e-mail: jinjiangchang0105@163.com). system. It makes a perfect combination between the accuracy of the traditional CAD and the flexibility of the technology curve-based modeling. The Constructed object is smooth NURBS curves and surfaces, rather than a straight line segment or polygon mesh surface. Rhino uses the trimmed free-form Trimmed free-form NURBS surfaces for showing curve shape accurately. It has curved projection characteristics, so that it can project curves on the entity and separate curves, then simplify the complex shape of the body. It is with the super ability to trim combination of objects of arbitrary curves, surfaces and solids. So it can be used as the transformation of a set of powerful 2D or 3D graphics or model documentation procedures and is worthy of a good design software [2], [3].

In summary, in the process of computer-aided design of military non-metal bulletproof helmet, in order to achieve better expression of the effect, it is suitable to choose the Rhino software in the design of the modeling phase of the helmet. Therefore, use the Rhino software to focus on modeling and model into other software and do the assisted modeling, texturing, advanced rendering and label the drawing size and so on.

The choice of software is closely linked to the quality of products and scientific research. Now we describe the using Rhino software for the design of military non-metal bulletproof helmet specifically.

\section{USING RHINO IN THE DESIGN OF HELMET}

Rhino is designed according to the product of the technical and functional requirements and through the operation of human-machine interaction to do product design ideas, Overall product design, technical design, analysis, calculation and so on.

The purpose of applying this technique to the design of military non-metal bulletproof helmet is to provide strong technical support and advanced design means for the development of relevant professional equipment for our military and to change the backwardness of the traditional design approach fundamentally, to improve the development level, to shorten the development cycle, to get good internal quality and appearance, to shorten the gap with foreign armies greatly.

In the past, in order to design any type of helmet, first of all, the designers must develop designs based on the types of tasks and targets, create the initial model. Then, there will be a lot of manual mapping and correction. After that we map out the engineering drawings. The following we should re-create the model and product samples, according to the size and data of the product on the drawings and do the 
laboratory performance testing, repeat modification, rendering steps according to test results. It takes a lot of time on the drawing board in the whole process. Besides the long design cycle, heavy workload, the most important thing is that this method can not describe and delivery the shape information of free curves and surfaces accurately. Also there are a lot of data loss and the inevitable human error in every aspect.

In the processing, because the shape information is controlled by the template and the way of manual operation, the machining accuracy is also difficult to ensure.

By adopting Rhino technology, we can uniquely define free type of curves and surfaces shape using mathematical method, construct the mathematical model of helmet products and molds accurately. It can fully reflect the design intent and ideas and lay a good foundation for future modification and processing.

\section{CREATE A SyStem PlatForm}

Rhino can create, edit, analyze, and translate NURBS curves and surfaces in the Windows systems without the restriction of complex degree, order number and size. Rhino also supports polygon meshes and point clouds. The features include: Uninhibited free-form 3D modeling tools; degree of precision in full compliance with the design, rapid prototyping, engineering, analysis and produce that required accuracy; compatible with other design, drafting, CAM, engineering, analysis, coloring, animation and illustration software; very easy to learn; high efficiency; no need of special hardware equipment; even in a general computer can perform.

The new AutoCAD software can help users complete the concept and detail design in a unified environment and create manage and share design work in one environment. The concept of design features is to let us find a suitable design in a faster and easier way and put such information as the basis of the design. AutoCAD is ideal for those professionals who carry out conceptual design manually, because it can speed up the design process. AutoCAD platform is with powerful and intuitive interface, so we can do the creation and modification of the appearance of graphics in a faster and easier way. Also, it provides some new features that provide more industry users with more explore design ideas in the early project design, provide faster feedback and more opportunities for design exploration [4]-[34].

Hardware:

AMD Athlon X2 3800+

2GB DDR SDRAM

$\mathrm{X} 800$

640GB Raid0

Software:

Rhinoceros 3.0 SR2

AutoCAD 2007

\section{The System Design THEORY}

The late 1980s, non-uniform rational B-spline methods (NURBS) can describe of the complex free curve accurately, at the same time, also resolve all simple curves and surfaces such as flat, cylindrical in properly, so that they can be coordinate in a mode and get unified management.

NURBS curves and surfaces have most nature of the B-spline curves and surfaces, as well as the following prominent characteristics are given:

1) Both standard analytic shape and accurate represent of free surface provides a common mathematical form. So that these two types of shape information can be stored in a unified database.

2) By manipulating the control points and weighting factors provide sufficient flexibility for a variety of shape design. The introduction of the weight factor as a substitute of the shape parameter in the geometric continuous spline curves and surfaces;

3) The stability and speed for commutating;

4) The geometric interpretation is obvious, and these who have a good knowledge of geometry, especially the designers of descriptive geometry knowledge can reap the particularly benefits;

5) The geometric matching techniques is strong;

6) Scaling, rotation, translation, shear and other operations is unchanged.

\section{Design AND Modeling OF Military Non-METAL BULLETPROOF HELMET}

\section{A. Prototyping and Data Acquisition}

In order to establish the mathematical model of helmet, the accurate measurement of the mold is needed. To this end, firstly, we must establish a uniform three-dimensional coordinate system which

Uses a head facial standard coordinate system. In this coordinate system, all data collection and measurement are completed.

\section{B. Critical Selection of Data Points and Surface Patches is Divided}

Taking into account the complex surface structure of the helmet, we cannot use a single spline or surface patches to fit. It shall be the divided appropriately then determine the value of the critical point and can be quantified. In this system, the helmet will be divided into shell section of the helmet, part of the brim, and the left and right auricle part. In which each auricular part are composed by the surface by the side of the auricle and ear helmet shell junction.

\section{Data and Graphical Input}

Draw a graphic in two-dimensional manner in each working plane, and then converted to three-dimensional coordinate system, Constructed three-dimensional structure of the model through appropriate editorial changes (shown in Fig. 1):

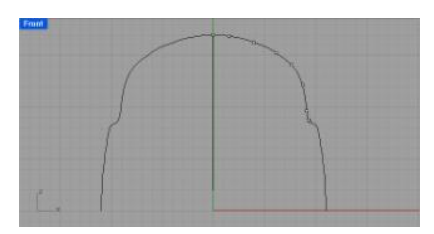

Fig. 1. Draw graphics in two-dimensional plane. 


\section{Curve and the Structure of the Framework Structure}

According to the coordinates of key points, draw two shape curves perpendicular to each other (shown in Fig. 2):

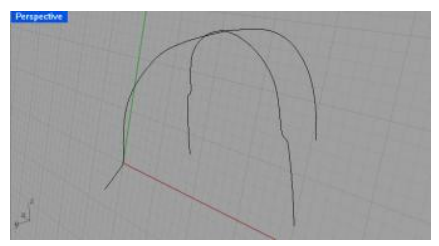

Fig. 2. Two shape curves perpendicular to each other.

Close hat with a circular curve (shown in Fig. 3):

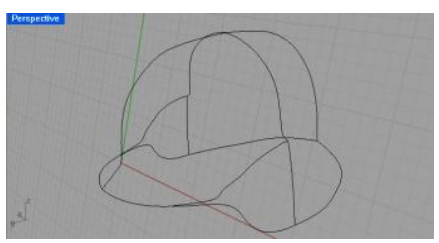

Fig. 3. Close hat with a circular curve.

Curve modification of the shape of the brim and ear (shown in Fig. 4):

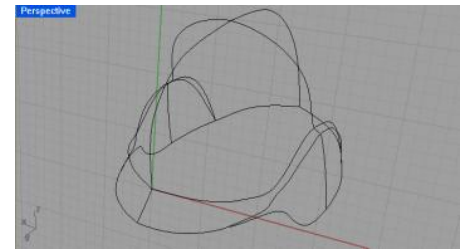

Fig. 4. Multi-curve modification brim and ear.

\section{E. Surface Fitting}

According to different occasions based on the drawing the curve before, using a rail loft, two-track loft and surface mesh methods to generate the helmet surface (shown in Fig. 5):

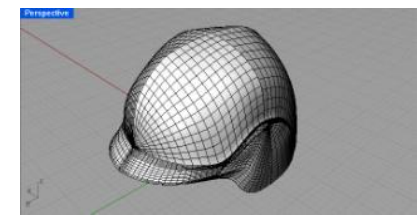

Fig. 5. Generate the helmet surface.

\section{F. Real-time Modification and Visual Display}

Surface integration on surface combined with the incomplete, Bonding sharp at the chamfer further improve the model (shown in Fig. 6):

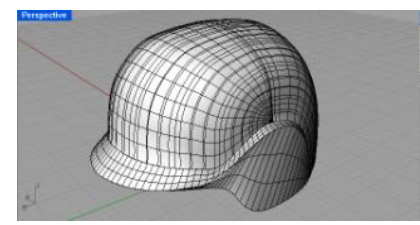

Fig. 6. Surface integration, bonding sharp at the chamfer.

Smooth the entire model, modify in detail, we complete the design (shown in Fig. 7):

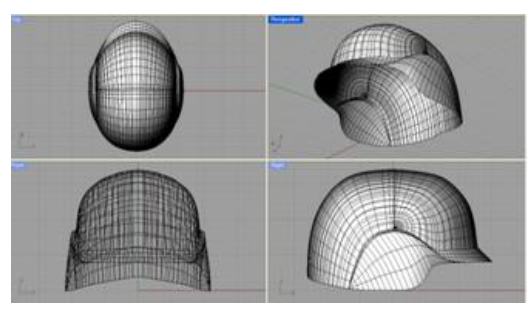

Fig. 7. Model smoothing, modified detail.

Engineering export to DWG format, transferred to AutoCAD 2007 dimension and other post-processing (shown in Fig. 8):

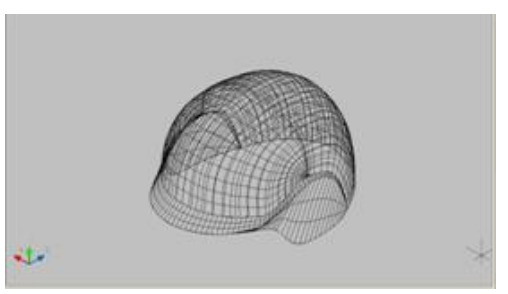

Fig. 8. DWG format drawing.

\section{CONCLUSION}

Using Rhino software and AutoCAD 2007 software combination, we get the Method of design of military non-metal bulletproof helmet. For those professionals in the conceptual design by hand, it can help us speed up the design process. Rhino software is a small, specifically designed to solve complex product surface modeling, 3D modeling software, have a high value on price, very low hardware requirements. So it can be freely used in the Windows environment. With powerful and intuitive interface, AutoCAD 2007 platform Can easily and fast creation and modification of the appearance of graphics. It also has some new features to make more sectors of the users to explore design ideas early in the project design. At last, it can provide faster feedback and more opportunities for design exploration.

The modelling ability of NURBS, vivid expression of texture and swift rendering speed should be laid more considerations when designing an ideal bulletproof helmet. Therefore, Rhino software is applied to model and then lead it into $3 \mathrm{~d}$ s max software to conduct texture mapping, senior rendering so as to reach the effect of complementation.

The tools should be mastered effectively in order to let the choice of software, quality of product and achievements in scientific research connected tightly. It is unknown that what kind of software can produce this product. The advantages and disadvantages of software can only be discovered when at work. The most commonly-used software includes $3 \mathrm{ds}$ max and Rhino in 3D design. We choose software according to their discrepancy which represents their merit and demerit. The effective software can have a swift, accurate and favorable design.

Applying the combination of Rhino and AutoCAD can make the professionals, who manually conduct conceptual design, accelerate to design non-metallic bulletproof helmets. Rhino is a 3D modeling software which mainly aims to solve the complicated surface modeling and can be applied to 
Windows without any restriction. AutoCAD owns a powerful visualized interface and can swiftly design and amend graphs.

The application of this technology on designing and processing non-metallic bulletproof helmets will provide powerful technological support and advanced processing method to produce helmets for our army. It can radically change the outdated traditional production method, improve production level, shorten production period and obtain favorable quality and appearance which will definitely narrow the discrepancy with the other armies.

The 2D design and 3D design can be exchanged interactively through the programming of $\mathrm{VC}++$ language on operating deck of Rhino3D. Therefore, it is possible for 3D graph of helmet to be changed to 2D graph of helmet and then directly put into production. The interactive exchange of 2D model and 3D model can provide a visualized effect and clear thought for designers to design helmets. Meanwhile, any design and modification of 3D and 2D model by designers can be showed on the visual interface of Rhino3D. It provides a great convenience for designers and lays a solid foundation for the CAD/CAM of helmet. Designers will have adequate freedom to return to the essence of design and concentrate on the structures and patterns of helmets. It will impact the CAD/CAM of helmet and bring great economic benefits for helmet manufacturing.

The application of virtual reality technology provides a new method for the design, research and evaluation of non-metallic bulletproof helmet. It changes the previous situation that helmet experiment is conducted separately when testing bulletproof property. However, when soldiers wear helmets, the response from soldiers is unknown if bullets hit their helmets. It improves the quality and speed of designing non-metallic bulletproof helmet and provides designers with clear design thought. It can integrate the design and manufacturing of helmet. Manufacturing can be conducted in the process of design and design can be conducted in the process of manufacturing. The comprehensive performance evaluation platform for non-metallic bulletproof helmet, which is based on virtual reality technology, will be beneficial to the design, research and development of equipment for our army's "Soldier System".

This technology will be applied to the design and processing of military non-metal bulletproof helmet, in order to change the backwardness of the traditional design of the mode of production in fundamentally, improve the development level, shorten the development cycle, get good internal quality and appearance, and shorten the gap with foreign armies greatly. In order to reduce costs, put into use in the combat effectiveness quickly, improve the efficiency of utilization, and highlight the economic benefits and great potential for development.

\section{REFERENCES}

[1] J. C. Jin and C. Wang, "An exploration on the compact level of the ballistic fabric of the military helmet and its ballistic performance," RJTA, vol. 12, pp. 50-57, May 2008.

[2] R. B. WANG, "Use Rhino 3dsmax optimizing the engineering drawing," Mechanical and Research Applications, vol. 3, pp. 77-79, Mar. 2004.
[3] X. F. MA, "Comparative analysis of product modeling renderings design software," Education and Vocational, vol. 18, pp. 63-64, Jun. 2004.

[4] H. W. Wang, AutoCAD2007 Machinery and Industrial Applications Classical Examples Tutorial, China Water Power Press, 2007.

[5] Z. Zheng, N. C. Yang, M. W. Shi, and K. L. Huang, "Researches and developments on hard antiballistic fiber composites," Journal of Materials Science \& Engineering, vol. 6, pp. 905-909, Nov. 2005.

[6] G. T. Zhou, "A compilation of specification for ballistic helmet and ballistic cloth of the USA army," Beijing: The Quartermaster Research Institute of the General Logistics Department of the CPLA, 1999.

[7] M. I. G. Bloor and M. J. Wilson, "Representin PDE surfaces in terms of B-splines," Computer-Aidted Design, vol. 222, no. 6, pp. 324-331, 1990.

[8] M. I. G. Bloor and M. J. Wilson, "Using partial defferential equations to generate free-from surfaces," Computer-Aided Design, vol. 22, no. 4, pp. 202-212, 1990

[9] W. Bohin, G. Farin, and J. Kahmann, "A survey of curte and surface methods in CAGD," Computer Aided Geometric Design, vol. 1, no. 1, pp. 1-60,1984

[10] G. Celniker and D. Gossard, "Deformable curve and surface finite-element for free-form shape design," Computer Graphics, vol. 25 , no. 4 , pp. $257-266,1991$

[11] G. Celniker and W. Welch, "Linear constrains for deformable B-spine surfaces," in Proc. Symposium on Interactive $3 D$ Graphics, pp. 165-170,1992

[12] J. E. C. Wick, D. R. Haumann, and R. E. Parcnt, "Layered construction for animated deformable charac-ters," Computer Graphics, vol. 23, no. 3, pp. 243-252, 1989

[13] S. E Chen and R. Parent, "Shape averaging and its applications to industrial design," IEEE Computer Graphics and Applications, vol. 9, no. 1 , pp.47-54, 1989

[14] C. de Boo, "On calculating with B-Splines," Journal of Approximation Theory, vol. 6, no. 1, pp.50-62, 1972

[15] G. Farin, "Trends in curve and sutface design," Computer-Aided Design, vol. 21, no. 5, pp. 293-296, 1989

[16] G. Farin, Curves and Surfaces for Computer aided Geometric Dseign: A Practical Guide, Academic Press, second edition, 1990.

[17] G. Farin, "From conics to NURBS: A tutorial and survey," IEEE Graphics and Applications, vol. 12, no. 5, pp. 78-86, Sept. 1992.

[18] D. R. Forsey and R. H. Bartels, "Hierarchical B-spline refinement," Computer Craphics, vol. 22, no. 4, pp. 205-212, 1988.

[19] B. R. Cossick, Hamilton's Principle and Physical Systems, Academic Press, New York and London, 1967.

[20] H. Karestucer, Finite Element Handbook, McCraw-Hill, New York, 1987.

[21] A. Kaul and J. Rossignac, "Solid interpolating deformations: Construction and animation of POPs," in Eurographics'91, F. H. Post and W. Barth, Eds., Amsterdam, North Holland, pp. 493-505, 1991.

[22] D. Metaxas and D. Terzopoulos, "Dynamic deformation of xolid primitives with constraints," Computer Graphics, vol. 26, 1989.

[23] M. Minoux, Mathematical Prongramming, Wiley, New York, 1986.

[24] H. P. Morteon and C. H. Sequon, "Functional optimization for fair surface design," Computer Graphics, vol. 26, no. 2, pp.167-176, 1992.

[25] A. Pentland and J. Williams, "Good vibrations: Modal dynamics for graphics and animation," Computer Graphics, vol. 23, no. 3, pp. 215-222, 1989.

[26] L. Piegl, "Modifying the shape of rational B-splines," Computer-Aided Design, Part 1: cutves, vol. 21, no. 9, pp. 528-546, 1989.

[27] L. Piegl, "Modifying the shape of rational B-sphines," Computer-Aided Design, Part 2: surfaces, vol. 21, no. 9, pp. 538-546, 1989.

[28] L. Piegl, "On NURBS: A survey," IEEE Computer Graphics and Applicatons, vol. 11, no. 1, pp. 55-71, Jan. 1991.

[29] L. Piegl and W. Tiller, "Curve and surface constructions using rational B-splines,” Computer-Aided Design, vol. 19, no. 9, pp. 485-525, 1992.

[30] J. Platt, "A generalization of dynamic constraints," CVGIP: Graphical Models and Lmage Processing, vol. 54, no. 6, pp. 516-525, 1992.

[31] J. Platt and A. Barr, "Constraints methods for flesible models," Computer Graphics, vol. 22, no. 4, pp. 279-288, 1988.

[32] W. Press. B. Flanney. S. Teukolsky, and W. Verttering, Nemerical Recipes: The Art of Scientific Comput., Ing, Cambridge University Press, Cambedge 1986.

[33] L. L. Schumaker, "Fitting surfaces to scattered data," in Apporximation Theory II, G. G. Lorentz, C. K. Chui, and L. L. Schumaker, Eds., Academic Press, New York, pp. 203-267, 1976.

[34] T. W. Sederberg, P. Gao, G. Wang, and H. Mu, "2-D shape blending: An intrinsic solurin to the vertex path prob-lem," in Proc. the Computer 
Graphics Proceedings, Annual Conference Series, ACM Siggraph'93(Anaheim, CA, Aug.1993), 1993, pp. 15-18.

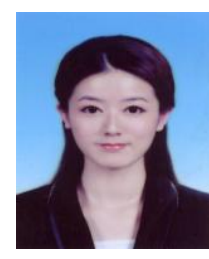

Haixin Jin was born on September 11, 1986, and now she lives in Beijing. She has achieved the degree of doctor of arts, master of art and bachelor of arts from Beijing Normal University in 2014, 2011 and 2008 respectively.

Dr. Jin is a member of Info Design Committee of China Industrial Association and has participated in the Fund Project of National Science and Technology Supporting Plan (2013BAH37F00). She now is an assistant researcher in postdoctoral station in the school of design of Tsinghua University and mainly engages in exhibition design. She has issued paper: Jin Haixin, A Brief Introduction to the Intertextuality between Film Narratology and Exhibition, Zhuangshi, vol. 12, pp. 39-41, December, 2014. In the future, she will focus on the research of material design.

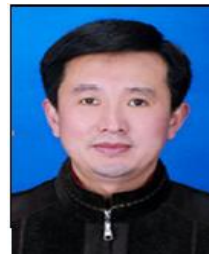

Jiangchang Jin was born on January 5, 1960, and now he lives in Shenzhen. He got the doctor of science from Huazhong University of Science and Technology in 2012, master of science from China Textile University in 1993 and bachelor of science from Wuhan Textile University in 1982. Now he is an advanced engineer in Shenzhen Academy of Metrology \& Quality Inspection and mainly engages in material inspection. He has issued paper: Jin Jiangchang \& Wang Chang, Discussion on Choosing Fabric Tightness Index to Design Ballistic Fabric, Science and Technology Innovation Herald, 2008, vol. 73, no. 1, pp. 71-74. In the future, he will focus on the research of quality inspection and material design. 